\title{
Re-examining sustained attention deficits as vulnerability indicators for schizophrenia: Stability in the long term course
}

\author{
Shi K. Liu ${ }^{\text {a }}$, Ming-Hsien Hsieh ${ }^{\text {a }}$, Tzung J. Hwang ${ }^{\text {a }}$, Hai-Gwo Hwu ${ }^{\text {a,b }}$, \\ Shih-Cheng Liao ${ }^{\mathrm{a}}$, Sheng-Hsiang Lin ${ }^{\mathrm{b}}$, Wei J. Chen ${ }^{\mathrm{a}, \mathrm{b}, *}$ \\ a Department of Psychiatry, National Taiwan University Hospital and College of Medicine, \\ National Taiwan University, 7 Chung-Shan South Road, Taipei 100, Taiwan \\ ${ }^{\mathrm{b}}$ Institute of Epidemiology, College of Public Health, National Taiwan University, 1 Jen-Ai Road, Section 1, Taipei 100, Taiwan
}

Received 17 June 2005; received in revised form 5 April 2006; accepted 28 June 2006

\begin{abstract}
This study examined the longitudinal patterns in the sustained attention deficits detected by the Continuous Performance Test (CPT) and the factors influencing such changes in consecutively admitted schizophrenia patients $(n=224)$ followed up for 4-7 year. Exploratory growth mixture modeling analyses of subjects' CPT performances over successive follow-ups revealed that three major (accounting for $92.8 \%$ ) plus one minor subgroups could be delineated. Subgrouping was then performed on a subsample of 104 subjects who had at least 3 times of CPT data. Based on subjects' adjusted $z$ score of the test sensitivity index $d^{\prime}$ derived from comparing with a community sample, patients were divided into three subgroups: no impairment $(\geqslant-1)$, moderate impairment $(-2.5$ to -1$)$, and severe impairment $(<-2.5)$. The trajectory taken by individual patient was analyzed according to the initial subgroup status and subsequent changes, controlling for relevant basic and clinical characteristics. Both growth mixture modeling and subgroup status analyses found that around one third of those with severe impairment at baseline showed persistent severe impairment. Those with no impairment were stable and exhibited least tendency for further performance deterioration. Those with moderate impairment tended to fluctuate markedly, mainly towards the better rather than the worse. Previous subgrouping status and concurrent task-taking strategy predicted the performance subgroup status at follow-ups, while clinical symptoms and disease course factors did not. We concluded that there is substantial heterogeneity in schizophrenia patients' long term pattern in sustained attention deficits and those with severe impairment might represent a subgroup with stable vulnerability to schizophrenia.
\end{abstract}

(c) 2006 Elsevier Ltd. All rights reserved.

Keywords: Schizophrenia; Continuous performance test; Sustained attention; Longitudinal study; Growth mixture modeling; Vulnerability indicator

\section{Introduction}

Sustained attention deficits measured by the Continuous Performance Test (CPT) (Rosvold et al., 1956) have been considered as potential vulnerability indicators for schizophrenia (Chen and Faraone, 2000; Cornblatt and Keilp, 1994; Keri and Janka, 2004). They were demonstrated in schizophrenic patients across illness stages (Asarnow and

\footnotetext{
* Corresponding author. Address: Institute of Epidemiology, College of Public Health, National Taiwan University, 1 Jen-Ai Road, Section 1, Taipei 100, Taiwan. Tel.: +8862 23123456x8360; fax: +886223560840.

E-mail address: weijen@ha.mc.ntu.edu.tw (W.J. Chen).
}

MacCrimmon, 1981; Nuechterlein and Dawson, 1984) and remained relatively unchanged despite fluctuations in clinical symptoms, in contrast to those manifested by affective disorders (Liu et al., 2002; Nuechterlein et al., 1994). In addition, the performance deficits were not amendable to treatment with neuroleptics when more difficult CPT versions were used (Finkelstein et al., 1997; Hong et al., 2002; Liu et al., 2000). People at high risk of schizophrenia, such as relatives of schizophrenia patients, were found to have worse CPT scores than normal controls (Egan et al., 2000; Mirsky et al., 1995; Saoud et al., 2000) and the CPT deficits were associated with both clinical and psychometric schizotypes (Chen et al., 1997; Laurent et al., 
2000; Roitman et al., 1997). The differential longitudinal changes in CPT performance thus might be indicative of the nature of the underlying vulnerability. For those schizophrenia patients in whom the genetic vulnerability plays a major role, their sustained attention impairments will be more severe and stable in the long-term course; otherwise, the severity of the impairments will be determined more by state-dependent factors, such as clinical states and treatment-related side effects, hence tend to fluctuate over the course (Chen and Faraone, 2000; Nuechterlein et al., 1994).

Despite such important theoretical implication, the longitudinal stability of CPT deficits in schizophrenic patients over the long-term course was seldom examined. Past follow-up studies were limited mainly to weeks or months after the immediate postpsychotic phase, rendering it premature to extrapolate the results to longer period (Epstein et al., 1996; Finkelstein et al., 1997; Hong et al., 2002; Liu et al., 2000; Nuechterlein et al., 1992). Indeed, a few studies with follow-up durations beyond one year did report improvements in the CPT deficits, despite their limited sample size (Cosway et al., 2002; Olbrich et al., 2001). However, most of the previous studies did not control for possible confounding factors of CPT performances, such as demographic characteristics, clinical symptom, medication, and treatment-related side effects. In addition, past follow-up studies approached the stability issues solely by analyzing the changes in the group means across different time points (Cosway et al., 2002; Epstein et al., 1996; Finkelstein et al., 1997; Hong et al., 2002; Liu et al., 2000; Nuechterlein et al., 1992; Olbrich et al., 2001), which might have masked the individual differences in the trajectories.

In this study, two major analytic strategies were undertaken to examine the issue of longitudinal stability in the CPT performance of schizophrenia patients with 4-7 years of follow-up. Analysis 1 was to explore whether there were indeed subgroups within schizophrenia through data-driven growth mixture modeling, hence to provide information about the optimal number of the subgroups as well as the characteristics of each subgroup's trajectories. Analysis 2 was to testify the hypothesis that CPT performance subgroups categorized according to their baseline CPT performance had different longitudinal stability over the follow-up periods. The longitudinal stability was defined in terms of the tendencies of the subjects to remain in their original subgroup status and was further analyzed with control for potential confounders to disentangle the effects of state-dependent factors from those of the vulnerability indicators.

\section{Methods}

\subsection{Subjects}

The study subjects were schizophrenic patients participating in two serial prospective follow-up studies for studying longitudinal course of psychopathological and neuropsychological manifestations of schizophrenia in Taiwan. The first study spanned from July 1993 to June 1998 and the second study was an extension of the first with the same study design from July 1998 to December 2001 (Chang et al., 2002; Hwu et al., 2002). Both were hitherto referred to as the Taiwan Psychopathology Study of Schizophrenia. During the enrolling period, one university and two university-affiliated hospitals recruited 234 consecutively admitted schizophrenia inpatients after written informed consent was obtained. The diagnosis was made according to the DSM-IV diagnostic criteria of schizophrenia tentatively at admission by senior attending psychiatrists. In addition, interview in person using the Chinese version of the Diagnostic Interview for Genetic Studies (DIGS) (Chen et al., 1998b; Nurnberger et al., 1994) by trained research assistants was used to collect all available information, such as care-giver report and previous medical records from other hospitals. The diagnoses were subsequently confirmed at discharge by independent assessments of three senior psychiatrists, based on catamnestic data and psychopathological manifestations during index admission. Among the 234 subjects, 6 (2.5\%) had inconsistent diagnoses and final diagnoses were reached through consensus meeting. Exclusion criteria were a history of electroconvulsive therapy in previous 6 months, mental retardation, trauma-related loss in consciousness for more than $30 \mathrm{~min}$, psychoactive substance abuse, or physical illness that might cast doubt on the diagnosis. The studies were approved by the Institutional Review Boards of the participating hospitals.

Although the main interest of this study was the changes within individual schizophrenic patients, we were still keen to know the magnitude of impairments in these patients compared to normal subjects. Hence a representative sample of community subjects was included as the norm. The normal comparison subjects were systematically sampled from the 1993 and 1994 voter lists of Chinshan Township, north of Taipei (Chen et al., 1998a). A total of 345 subjects, about $65 \%$ of selected local residents, were successfully tested. Subjects who had a diagnosis of psychosis according to the outpatient records at Chinshan Health Station or stroke were excluded from the normative data.

\subsection{Measures}

\subsubsection{Clinical assessments and psychopathology measures}

Baseline information regarding age at onset, duration of illness, previous medication and hospitalization histories were collected systemically. The Chinese version of the Positive and Negative Syndrome Schedules (PANSS) (Kay et al., 1987), which was found to have sufficient inter-rater reliability (Cheng et al., 1996) was used by trained senior research psychiatrists to assess clinical psychopathology with the following time frame: admission, discharge, 3 months, 6 months, 12 months, and then on a yearly basis. 
Our previous study on the symptom dimensions of the PANSS revealed a five-factor structure (Hwu et al., 2002) and the compositions of these factors were largely in accordance with those from other studies (Mass et al., 2000). As the symptom dimensions were more closely related to neurocognitive performances than the original PANSS sub-scales (Liu et al., 1997), we averaged the summed-up individual PANSS item ratings within the same factor to yield five mean factor scores, i.e., Negative, Disorganization, Positive, Depression, and Excitement. Subsequent analyses on clinical psychopathology were all done on these mean factor scores.

\subsection{2. $C P T$}

The subjects were assessed with the CPT, version 2.20, operated on a Sunrise Systems machine (Pembroke, Mass.). The procedure and its test-retest reliability have been described in detail elsewhere (Chen et al., 1998a). Briefly, numbers from 0 to 9 were randomly presented for $50 \mathrm{msec}$ each, at a rate of one per second for two sessions (undegraded and 25\% degraded). A total of 331 trials, $34(10 \%)$ of which were target stimuli (the number 9 preceded by the number 1), were presented over $5 \mathrm{~min}$ for each session. During the second session (25\% degraded session), a pattern of snow was used to toggle background and foreground so that the image was visually distorted. Two signal-detection indices of CPT performance - sensitivity $\left(d^{\prime}\right)$ and response criterion (natural logarithm of $\beta[\ln \beta]$-were derived from the hit rate (probability of response to target trials) and false-alarm rate (probability of response to nontarget trials) (Davies and Parasuraman, 1982; Swets, 1973). The sensitivity index $d^{\prime}$ measures an individual's ability to discriminate target stimuli from nontarget stimuli, while the response criterion index $\ln \beta$ measures the amount of perceptual evidence that the person requires to identify a stimulus as a target, hence an indicator of patient's task-taking strategy. Variables extraneous to the intended comparison of sensitivity, such as differences in level of motivation or cooperativeness, would yield differences in $\ln \beta$ rather than in $d^{\prime}$.

The baseline CPT assessments were administered after patients' clinical conditions stabilized enough. Of the initial 234 patients enrolled in the Taiwan Psychopathology Study of Schizophrenia, 187 completed the baseline CPT assessment. About 4 years later, CPT assessments were repeated on a yearly basis for three times. Overall, each subject was scheduled to have 4 times CPT assessments over the entire study period.

\subsection{Data analysis}

\subsubsection{Analysis 1: Growth mixture modeling}

To explore whether subgroups with distinct trajectories of changes in CPT performances could be delineated within current sample, growth mixture modeling was used to analyze data from those patients who had at least one CPT assessment during the entire follow-up period (224 out of the 234 patients enrolled) using MPlus software (Muthen and Muthen, 2005). Subjects not included did not differ in basic demographic characteristics from those included for this analysis. The number of subgroups was not set a priori. Rather, models with successive numbers of subgroups were compared against each other, starting from a model with 2 subgroups. The model with most significant difference with its previous model of one subgroup less was selected, based on the likelihood ratio test with significance level set at $p<0.05$. In addition, on the basis of Bayesian Information Criteria (BIC), the model with the lowest BIC was preferred (Muthen et al., 2002).

\subsubsection{Analysis 2: Subgroup status change}

We then subgrouped patients according to their baseline CPT performance to examine whether different subgroups had different longitudinal stability over the follow-up periods. Of the entire 234 patients, 104 patients completed more than 3 times CPT assessments, 80 completed one or two CPT assessments and 40 patients did not complete any CPT test. Only subjects with CPT performance data for at least three time points were included for this analysis. For those with 4 times of CPT assessments $(n=58)$, in addition to the baseline and the last assessments, one additional CPT assessment was chosen between the remaining two to make the CPT test intervals more evenly distributed. The mean interval between the baseline assessment and the first follow-up was 1213.9 ( $\mathrm{SD}=390.8$ ) days, between the first and the second follow-up was $597.0(\mathrm{SD}=182.1)$ days, and between the baseline and the second follow-up was $1807.1(\mathrm{SD}=400.5)$ days.

To better understand their magnitude of deficits compared to normal comparisons, patients' CPT raw scores were standardized against the community norm with adjustment for demographic features to provide adjusted $z$ scores (Chen et al., 1998a). Since age, sex, and educational level have been demonstrated to influence the CPT performance in the community sample, the predictive score of a subject was calculated by using the regression coefficients obtained from the regression of the scores on the three covariates among the 345 community subjects. The difference between the raw score and the predictive score was then standardized by the root mean error of the regression and was defined as the adjusted $z$ score of the subject. The adjusted $z$ scores thus provide an estimate of the magnitude of deviation from the community norm.

As our previous studies demonstrated that the degrees of CPT performance impairments in schizophrenic probands were related to the performances in unaffected relatives (Chen et al., 2004; Chen et al., 1998b), we chose the interface values of the three-component distribution of -1 and -2.5 (adjusted $z$ score of $d^{\prime}$ ) from our previous admixture analysis on the CPT performances in both the non-psychotic relatives of schizophrenia patients and normal comparison subjects (Chen et al., unpublished data) as cut-off points. Intriguingly, these cut-off points were compatible with the results of growth mixture modeling. 
Accordingly, patients with an adjusted $z$ score of $d^{\prime}>-1$ were categorized as the group with no impairment (NI), those with an adjusted $z$ score $<-2.5$ as the group with severe impairment (SI), and those with an adjusted $z$ score in between as the group with moderate impairment (MI).

In assessing the change in CPT performance grouping status across time, two measures of stability were calculated: prospective consistency and retrospective consistency. Prospective consistency equals to the proportion of individuals in a group at time 1 remaining in the same group at time 2 . In contrast, retrospective consistency equals the proportion of individuals in a group at time 2 belonging to the same group at time 1 .

The stability of the CPT-based subgrouping was further evaluated by exploring whether a later subgroup assignment could be predicted by a previous CPT performance. As the SI group is our major interest, we collapsed the NI and MI groups into a single group to contrast with the SI group and logistic regression analysis with the CPT group status (severe vs. non-severe impairment) at time 2 as dependent variable and previous group assignment at time 1 as the independent was conducted separately for the three different follow-up time frames. The extraneous factors that might have affected the CPT performances, including concurrent $\ln \beta$ at time 2, age at onset, duration of illness, interval between the two CPT assessments, and the 5 mean PANSS-CH symptom factor scores, were included as covariates. The Wald test was used to estimate the significance of the regression coefficients. The significance for all statistical test was set at a $p$ value smaller than 0.05 without correction for multiple comparisons.

\section{Results}

The growth mixture modeling analysis of the 224 subjects' changes in undegraded CPT $d^{\prime}$ revealed that a 4-subgroup model best fitted the data (Fig. 1). The model with 4 subgroups had a better fit than the model with 3 subgroups ( $p=0.037$ for the likelihood ratio test; adjusted $\mathrm{BICs}=1959.8$ and 1975.1, respectively). The number of subjects $(\%)$ in each subgroup was $62(27.6 \%), 17(7.8 \%)$, $91(40.7 \%)$, and $54(24.0 \%)$ with the corresponding mean $d^{\prime}$ 's being $0.08,3.27,3.43$, and 0.92 , respectively, indicating that the 4 subgroups were distinguished by their trajectories of changes as well as their initial severity of impairments. Overall, the three main subgroups all showed a trend toward improvements in CPT $d^{\prime}$ during the successive follow-ups, whereas the smallest subgroup had a deteriorating course in CPT performance despite its initial good performance. Because the smallest subgroup comprised only $7.8 \%$ of total cases, the major trends for the trajectories of changes within the entire sample were better represented by the other 3 major subgroups. Similar results were obtained for the degraded CPT (data not shown).

The 104 subjects included for analysis 2 were of relatively young ages and medium disease chronicity with an even distribution in sex (Table 1). Although these 104

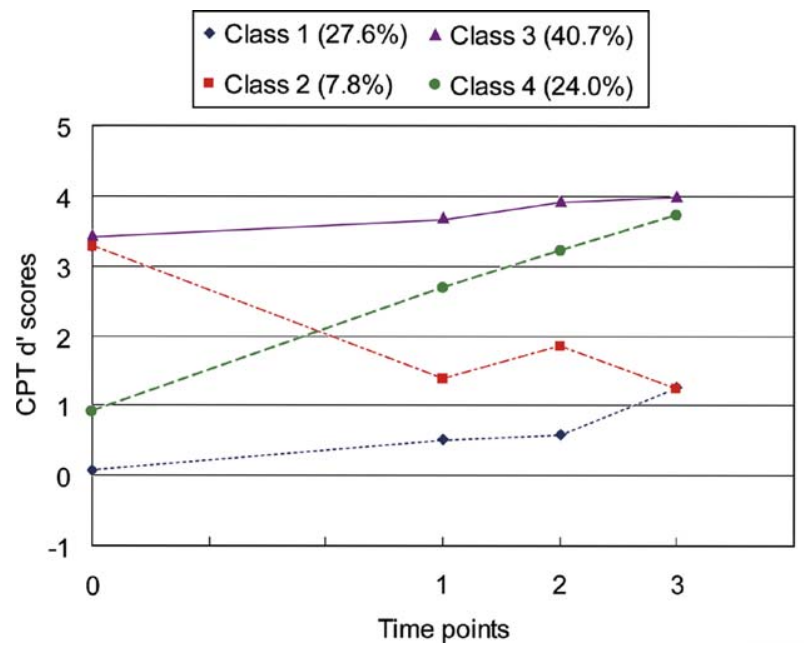

Fig. 1. Trajectories of the CPT performances of schizophrenia patients $(n=224)$. Time point $0=$ baseline, $1-3=$ first to third follow-up.

patients comprised only $44.4 \%$ out of the 234 patients enrolled, univariate analyses comparing them with those without CPT data $(n=49)$ or those with less than three CPT assessments $(n=80)$ did not reveal any significant differences in term of demographic characteristics, clinical course variables, and baseline symptom factor scores and CPT performances.

As the degraded CPT performance scores were highly correlated with the undegraded ones and the pattern of changes was similar to that of the undegraded ones too, only the results of the undegraded CPT were reported (Table 2). Patients' CPT performances as a group at the three assessments were all substantially impaired since their mean adjusted $z$ scores of $d^{\prime}$ were significantly below those of the community sample. In contrast, the mean adjusted $z$ scores of the $\ln \beta$ were not statistically deviant from the community norm. As a group, subjects' $d^{\prime}$ and $\ln \beta$ improved significantly across the follow-up period, no matter the raw scores or the adjusted $z$ scores. The intraclass correlation coefficient for the three CPT assessments was $0.55(F=4.73[\mathrm{df}=103,206], p<0.0001 ; 95.00 \%$ C.I.: 0.45-0.65), indicating that the CPT performances over the time points were moderately correlated. Antipsychotic dosage did not correlate with any CPT indices and patients receiving typical antipsychotics did not differ from those receiving atypical ones in their CPT performances.

At baseline, the CPT performance subgroups NI, MI and SI comprised $25 \%, 20 \%$, and $55 \%$ of total subjects, respectively (Table 3). After 4-7 years, the corresponding fractions changed to $49 \%, 23 \%$, and $28 \%$, indicating an overall trend toward improvement in CPT performances. However, the consistency indices demonstrated the different trajectories of changes among the three groups. The NI subgroup showed least tendency to shift out, as revealed by their consistently high prospective consistencies across all the three time frames (58-90\%), but substantial case shifting-in in the longer follow-ups, as indicated by the 
Table 1

Demographic and clinical course characteristics, psychopathological manifestations, and CPT performance indices of patients recruited for the Taiwan Psychopathology Study of Schizophrenia, 1993-2001

\begin{tabular}{|c|c|c|c|}
\hline \multirow[t]{2}{*}{ Variable } & \multirow{2}{*}{$\begin{array}{l}\text { Included in the subgrouping analyses }{ }^{\mathrm{a}}(n=104) \\
N(\%)\end{array}$} & \multicolumn{2}{|c|}{ Not included in the subgrouping analyses } \\
\hline & & $\begin{array}{l}\text { CPT test no. } \leqslant 2(n=80) \\
N(\%)\end{array}$ & $\begin{array}{l}\text { CPT not assessed }(n=49) \\
N(\%)\end{array}$ \\
\hline Male & $53(50.5)$ & $41(51.4)$ & $23(49.9)$ \\
\hline Being first admission & $14(13.3)$ & $2(4.1)$ & $13(16.5)$ \\
\hline \multirow[t]{2}{*}{ Being on job in the previous one year } & $38(36.1)$ & $32(40.0)$ & $15(30.6)$ \\
\hline & Mean $(S D)$ & Mean $(S D)$ & Mean $(S D)$ \\
\hline Age & $30.4(7.0)$ & $31.7(8.2)$ & $32.5(7.4)$ \\
\hline Education (year) & $11.2(2.8)$ & $10.9(3.0)$ & $10.7(3.0)$ \\
\hline Age at onset & $21.8(6.2)$ & $24.0(7.1)$ & $23.4(6.1)$ \\
\hline Duration of illness (year) & $8.6(5.9)$ & $7.6(6.1)$ & $9.1(4.5)$ \\
\hline No. of previous admission & $2.5(2.1)$ & $2.3(2.1)$ & $2.6(2.5)$ \\
\hline \multicolumn{4}{|l|}{ Mean PANSS factor scores at baseline } \\
\hline Negative & $2.5(0.8)$ & $2.6(0.9)$ & $2.7(0.9)$ \\
\hline Disorganization & $1.9(0.9)$ & $2.2(1.0)$ & $2.0(1.0)$ \\
\hline Positive & $2.7(1.0)$ & $2.3(1.1)$ & $2.8(1.1)$ \\
\hline Depression/anxiety & $1.6(0.8)$ & $1.7(0.8)$ & $1.8(0.7)$ \\
\hline Excitement & $1.7(1.0)$ & $1.8(1.3)$ & $1.8(1.0)$ \\
\hline \multicolumn{4}{|l|}{ Baseline undegraded CPT $d^{\prime}$} \\
\hline Raw score & $2.1(1.8)$ & $1.8(1.7)$ & - \\
\hline Adjusted $z$ score & $-3.2(2.5)$ & $-3.6(2.3)$ & - \\
\hline
\end{tabular}

All comparisons among the three groups were not statistically significant at the 0.05 level.

a Patients receiving three CPT evaluations or more were included.

relatively low retrospective consistencies $(39 \%)$. For the SI subgroup, the high retrospective consistencies across all three time frames $(72-90 \%)$ indicated that few new cases moved into the SI subgroup. In contrast, the prospective consistencies of the SI subgroup decreased as the followup intervals lengthened. For the shorter interval from 1st follow-up to 2nd follow-up, 61\% remained within the SI subgroup, while for the longest interval from baseline to 2nd follow-up, the percentage decreased remarkably to $39 \%$. Thus, a substantial proportion of the initial SI subjects had improved performances at long-term follow-up. As to the MI subgroup, their prospective and retrospective consistencies across all time frames remained very low,

Table 2

Subjects' CPT performance indices and antipsychotic use at baseline and subsequent follow-ups $(n=104)$

\begin{tabular}{|c|c|c|c|}
\hline & Baseline & 1st follow-up & 2nd follow-up \\
\hline & Mean (SD) & Mean (SD) & Mean (SD) \\
\hline \multicolumn{4}{|l|}{ Undegraded CPT indices } \\
\hline Raw score of $d^{\prime a}$ & $2.1(1.8)$ & $2.6(1.7)$ & $3.1(1.6)$ \\
\hline Raw score of $\ln \beta^{\mathrm{b}}$ & $1.4(1.8)$ & $1.8(1.5)$ & $2.1(1.3)$ \\
\hline Adjusted $z$ score of $d^{\prime a}$ & $-3.2(2.5)$ & $-2.4(2.4)$ & $-1.7(2.1)$ \\
\hline Adjusted $z$ score of $\ln \beta^{\mathrm{b}}$ & $-0.8(2.2)$ & $-0.4(1.8)$ & $0.0(1.5)$ \\
\hline Antipsychotic dosage $^{\mathrm{c}}$ & $902.3(592.2)$ & $826.6(515.4)$ & $815.6(536.8)$ \\
\hline \multicolumn{4}{|c|}{$\begin{array}{l}{ }^{\mathrm{a}} p<0.001 \text {, paired- } t \text { test, pairwise comparisons between successive } \\
\text { assessments. } \\
{ }^{\mathrm{b}} p<0.05 \text {, paired- } t \text { test, pairwise comparisons between successive } \\
\text { assessments, except comparison between } 1 \text { st and } 2 \text { nd follow-ups. } \\
{ }^{\mathrm{c}} \text { Chlorpromazine equivalents. }\end{array}$} \\
\hline
\end{tabular}

hence the most unstable among the three groups in terms of the case shifting tendency.

The determinants of later CPT performance subgroup assignment were explored by the logistic regression analyses across the three follow-up time frames (Table 4). Previous CPT grouping (by $d^{\prime}$ level) and concurrent CPT task-taking strategy $(\ln \beta)$ were the major determinants for current CPT group assignment when factors extraneous to CPT were controlled for. Previous assignment in the SI subgroup as well as poorer concurrent $\ln \beta$ presented a higher risk to be classified as SI at follow-up (odds ratio ranged from 2.24 to 9.10 for previous CPT subgrouping and from 2.13 to 2.91 for $\ln \beta$, respectively). The diseaseassociated variables (duration of illness, age at onset, interval between follow-ups, and antipsychotic dosage) and clinical symptom dimensions (negative, disorganization, positive, depressive, and excitement factor) did not contribute significantly to later subgroup assignment. Adding other disease course characteristics, such as the number of rehospitalization and antipsychotic usage, did not alter the results.

\section{Discussion}

So far as we know, this is the longest follow-up study with multiple assessments of schizophrenia patients' sustained attention deficits, enabling us to address the issue of longitudinal stability beyond the immediate post psychotic phase. Overall, our results revealed a general tendency to improve in sustained attention during the entire follow-up period and about $1 / 3$ of the schizophrenia 
Table 3

Distribution of the undegraded CPT performance grouping during successive follow-ups

\begin{tabular}{|c|c|c|c|c|c|c|}
\hline \multirow[t]{2}{*}{ Initial grouping $^{\mathrm{a}}$} & \multirow[t]{2}{*}{$N(\%)$} & \multicolumn{3}{|c|}{ Subsequent grouping } & \multicolumn{2}{|c|}{ Consistency in grouping $(\%)$} \\
\hline & & $\mathrm{NI}$ & MI & SI & Prospective & Retrospective \\
\hline Baseline to 1st follow-up & $104(100)$ & $38(37)$ & $23(22)$ & $43(41)$ & & \\
\hline No impairment (NI) & $26(25)$ & 15 & 7 & 4 & 57.6 & 39.5 \\
\hline Moderate impairment (MI) & $21(20)$ & 10 & 3 & 8 & 14.3 & 13.0 \\
\hline Severe impairment (SI) & $57(55)$ & 13 & 13 & 31 & 54.4 & 72.1 \\
\hline Baseline to 2nd follow-up & $104(100)$ & $51(49)$ & $24(23)$ & $29(28)$ & & \\
\hline No impairment & $26(25)$ & 20 & 4 & 2 & 76.9 & 39.2 \\
\hline Moderate impairment & $21(20)$ & 11 & 5 & 5 & 23.8 & 20.8 \\
\hline Severe impairment & $57(55)$ & 20 & 15 & 22 & 38.6 & 75.9 \\
\hline 1st follow-up to 2 nd follow-up & $104(100)$ & $51(49)$ & $24(23)$ & $29(28)$ & & \\
\hline No impairment & $38(37)$ & 34 & 3 & 1 & 89.5 & 66.7 \\
\hline Moderate impairment & $23(22)$ & 10 & 11 & 2 & 47.8 & 45.8 \\
\hline Severe impairment & $43(41)$ & 7 & 10 & 26 & 60.5 & 89.7 \\
\hline
\end{tabular}

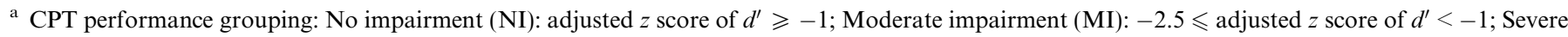
impairment (SI): adjusted $z$ score of $d^{\prime}<-2.5$.

Table 4

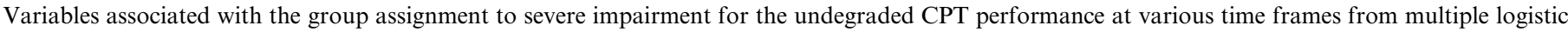
regression analysis ${ }^{\mathrm{a}}(n=104)$

\begin{tabular}{|c|c|c|c|c|}
\hline Time frame for the outcome & Predictors & $\beta(\mathrm{SE})$ & $p$ & Odds ratio $(95 \% \mathrm{CI})$ \\
\hline \multicolumn{5}{|l|}{ Baseline to 1st follow-up } \\
\hline \multirow[t]{2}{*}{$\left(r^{2}=0.46\right)$} & Previous CPT $d^{\prime}$ group & $0.81(0.38)$ & 0.03 & $2.24(1.54-3.29)$ \\
\hline & Concurrent CPT $\ln \beta$ & $0.75(0.23)$ & 0.001 & $2.13(1.68-2.66)$ \\
\hline \multicolumn{5}{|l|}{ 1st follow-up to 2 nd follow-up } \\
\hline \multirow[t]{2}{*}{$\left(r^{2}=0.64\right)$} & Previous CPT $d^{\prime}$ group & $2.20(0.63)$ & $<0.001$ & $9.01(4.81-16.95)$ \\
\hline & Concurrent CPT $\ln \beta$ & $0.75(0.25)$ & 0.003 & $2.71(2.23-2.72)$ \\
\hline \multicolumn{5}{|l|}{ Baseline to 2 nd follow-up } \\
\hline \multirow[t]{2}{*}{$\left(r^{2}=0.49\right)$} & Previous CPT $d^{\prime}$ group & $0.99(0.43)$ & 0.02 & $2.68(1.75-4.14)$ \\
\hline & Concurrent $\mathrm{CPT} \ln \beta$ & $1.07(0.26)$ & $<0.001$ & $2.91(2.25-3.78)$ \\
\hline
\end{tabular}

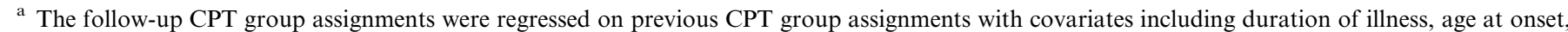

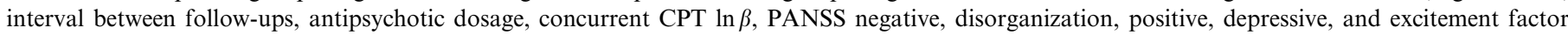
scores. Only covariates with significant regression coefficients $(p<0.05)$ were shown.

subjects with severe impairments in attention remained so through out the period. The correlations in the CPT performances across assessment time points were "good" but not "excellent" in terms of test-retest reliability, indicating that CPT performances changed considerably during a long follow-up period. The tendency to improve in the whole sample was supported by the data-driven growth mixture modeling analysis, in which three major subgroups could be demarcated by their trajectories and initial levels of impairments, as well as the subsequent subgrouping analyses. The patterns of shifting between subgroups might partly arise from the fact that the NI and SI subgroups changed subgroup status less than the middle group simply because that they each had only one direction in which to move, while patients in the MI subgroup could move either up or down in the distribution. However, the unanimous direction toward improvements made such an explanation less likely to account fully for the findings.

At first glance, the results did not seem to support CPT deficits as stable vulnerability indicators. However, it was notable that the proportion of patients with low perfor- mance over the follow-up period was $27.1 \%$ in the growth mixture modeling, which was comparable to the figure of $24.1 \%$ for those with severe impairments at baseline that remained severely impaired throughout the follow-up period. Moreover, the most important determinants of later poor CPT performances were found to be previous severe impairments, implying that CPT deficits with sufficient severity were not substantially influenced by extraneous factors and sufficed to predict their own further development even after long follow-up intervals. In this regard, severe CPT deficits probably reflected the core neurobiological vulnerability traits.

Some precaution must be pointed out, however. Deficits across multiple neurocognitive domains have been considered characteristic of schizophrenia (Flashman and Green, 2004). Their associations with obstetric complications (Cannon et al., 2002), structural brain changes and subtle neurological signs (Arango et al., 2000; Leask et al., 2002; Obiols et al., 1999) suggested probable neurodevelopmental origins (Cannon et al., 2003; Lawrie et al., 2001; Lewis and Levitt, 2002). In this regard, the observed persis- 
tent deficits on CPT might be one part of the static encephalopathy associated with early developmental insults (Hill et al., 2004). In addition, later disease- and treatment-related neurocognitive side effects might have also contributed to the sustained attention deficits. Indeed, when attention deficits alone were employed to predict the occurrence of schizophrenic spectrum disorders, unacceptable high false positive rate ensued (Keri and Janka, 2004), underscoring the part played by non-genetic factors. However, studies among nonpsychotic relatives of schizophrenia patients (Chen et al., 2004; Ross et al., 2000) found that the neurocognitive deficits per se were familial in these families. Our recent finding that siblings from multiplex families exhibited worse CPT performance than did their counterparts from simplex families (Tsuang et al., in press) provided further support for the proposition that impairment in sustained attention is a genetic vulnerability marker for schizophrenia. Further strategy to disentangle genetic from non-genetic factors on CPT deficits thus should focus on the dosage effect in the transmission pattern within families of those with persistent severe impairments, in contrast with that within less impaired patients' families. In addition, other signs of early brain injury and neurological dysfunction should be carefully sought and controlled for (Lawrie et al., 2001; Leask et al., 2002; Lewis and Levitt, 2002).

The substantial proportion of subjects with CPT performance within population norm was another distinct subgroup and could be considered as lying at the 'normal' end of the liability continuum. Neuropsychologically normal schizophrenia subjects have been a non-rarity and they demonstrated a clinical profile and disease course different from those with abnormal performances (Kremen et al., 2000; Palmer et al., 1997). Although there might still be some decline from premorbid level of performance (Kremen et al., 2000), these patients were nonetheless considered relatively, though not absolutely, devoid of the disease/genetic vulnerability-related decrements. In this regard, they could serve as internal contrast group within schizophrenia proper to disentangle the effects of clinical state-related and genetic-related factors, hence a powerful tool to explore the issue of genetic heterogeneity within schizophrenia.

In contrast to previous two subgroups, those with CPT performance at intermediate level showed marked instability during the long-term course, suggesting that their performances were mainly state-dependent. In this regard, the prospect that some major gene effects be found from this group is expected to be meager. In addition, since many recent pharmacological interventional studies on sustained attention deficits were carried out in patients with modest degree of impairments with short-term follow-up (Cornblatt et al., 1997; Epstein et al., 1996; Hong et al., 2002), the intrinsic instability of this group's short-term performances rendered the results as preliminary at best.

In logistic regression analyses, only two factors consistently emerged as significant predictors for CPT perfor- mance group assignment, i.e., previous CPT grouping status and concurrent response criterion $\ln \beta$, whereas variables of disease course and clinical symptoms did not. The results seemed to be inconsistent with previous findings that certain clinical symptom dimensions were associated with the CPT deficits, especially negative and thought/disorganization symptoms (Guillem et al., 2001; Liu et al., 1997; Mass et al., 2000; Nuechterlein et al., 1986; Stratta et al., 2000; Strauss, 1993). Several reasons might have accounted for this discrepancy. First, meta-analysis on the associations between CPT and psychopathology revealed low to moderate associations (weighted $r=-0.31$ ), with negative symptoms accounting for less than $10 \%$ of the variances in CPT $d^{\prime}$ (Nieuwenstein et al., 2001). Second, previous studies either were limited to cross-sectional analyses (Mass et al., 2000; Stratta et al., 2000) or examined only cross-temporal correlations between the two in a short period of time (Nuechterlein and Dawson, 1984). Although both sustained attention deficits and negative symptoms were enduring features of schizophrenia, it is still possible that they were determined by distinct pathophysiological mechanisms rather than a common underlying neurocognitive deficit (Tamminga et al., 1998). In this regard, the reported associations between negative symptoms and sustained attention deficits might not be an invariable phenomenon for all schizophrenia patients.

It was also noteworthy in current study that CPT performances were significantly influenced by the response strategy. Our past studies revealed that schizophrenia patients seemed to take a more lenient strategy when undertook a more difficult version of the CPT. In contrast, when an easier attentional task was administered, patients were less likely to disengage their attention and little effects on performances would be expected (Liu et al., 2000; Liu et al., 2002; Liu et al., 1997). In addition, $\ln \beta$ was found to be independent from the sensitivity index $d^{\prime}$ in community subjects, but the two indices exhibited a modest correlation in schizophrenia patients (Chen et al., 1998a). Thus, schizophrenia patients' deficits in CPT performances should be carefully examined in terms of the task-taking strategy, especially when more difficult versions with perceptual loads were used.

Limitations of this study should be kept in mind while interpreting the results. First, the lack of a longitudinal normal control group in this study made it difficult to determine whether the subgroup shifting could be found in normal subjects too as a result of natural tendency for fluctuations in CPT performances. However, there has been evidence that performances in multiple neurocognitive domains, in which attention was included, remained stable across assessments in normal subjects, hence not likely to account for the changes observed in schizophrenia patients (Heaton et al., 2001). Second, subjects included for analysis 1 and analysis 2 were different subpopulations of the total sample and the results might have been systemically biased. Nevertheless, comparisons in the demographic 
and clinical characteristics did not reveal significant differences between those included and not included for both analyses 1 and 2. Compatible results revealed by both analysis 1 and analysis 2 further indicated that the selection of subjects for analysis 2 might not have severely distorted the results. On the other hand, those without baseline CPT data were mainly from those unable to complete even the undegraded version, hence more likely to be severely impaired. The proportion of the SI subgroup thus might have been under-estimated. Hence, the associations between CPT deficits and negative symptoms might have been underestimated in this study.

In summary, this study demonstrates that there were distinct subgroups in schizophrenia patients that exhibited different trajectories in the long term course of sustained attention deficits, which might have important theoretical and practical repercussions for further research into the heterogeneity of schizophrenia. Despite the fluctuations, there is indeed a subgroup of severely impaired patients whose sustained attention deficits might represent a stable vulnerability indicator for schizophrenia.

\section{Acknowledgements}

Supported by grants from the National Health Research Institutes, Taiwan (DOH 88-HR-825; NHRI-GTEX89P825P; NHRI- EX90-8825PP; NHRI- EX919113PP; NHRI- EX92-9113PP) and National Science Council, Taiwan (NSC83- 0412-B-002-310, NSC84-2331B-002-187). The authors thank other participants in the Taiwan Psychopathology Study of Schizophrenia who helped in the recruitment and evaluation of schizophrenia patients, including Drs. Ching-Jui Chang, Hung-Jung Chang, Hai Ho, Ping-Ju Chang, Shi-Chin Guo, ShianYuan Lan, Su-Kuan Lin, Fu-Chuan Wei, and Joseph J. Cheng.

\section{References}

Arango C, Kirkpatrick B, Buchanan RW. Neurological signs and the heterogeneity of schizophrenia. American Journal of Psychiatry 2000;157:560-5.

Asarnow RF, MacCrimmon DJ. Span of apprehension deficits during the postpsychotic stages of schizophrenia: A replication and extension. Archives of General Psychiatry 1981;38:1006-11.

Cannon TD, van Erp TG, Rosso IM, Huttunen M, Lonnqvist J, Pirkola $\mathrm{T}$, et al. Fetal hypoxia and structural brain abnormalities in schizophrenic patients, their siblings, and controls. Archives of General Psychiatry 2002;59:35-41.

Cannon TD, van Erp TG, Bearden CE, Loewy R, Thompson P, Toga $\mathrm{AW}$, et al. Early and late neurodevelopmental influences in the prodrome to schizophrenia: contributions of genes, environment, and their interactions. Schizophrenia Bulletin 2003;29:653-69.

Chang C-J, Chen WJ, Liu S-K, Cheng JJ, Ou Yang W-C, Chang H-J, et al. Morbidity risk of psychiatric disorders among the first degree relatives of schizophrenic patients in Taiwan. Schizophrenia Bulletin 2002;28:379-92.

Chen WJ, Faraone SV. Sustained attention deficits as markers of genetic susceptibility to schizophrenia. American Journal of Medical Genetics (Seminars in Medical Genetics) 2000;97:52-7.
Chen WJ, Hsiao CK, Lin CCH. Schizotypy in community samples: The three-factor structure and correlation with sustained attention. Journal of Abnormal Psychology 1997;106:649-54.

Chen WJ, Hsiao CK, Hsiao L-L, Hwu H-G. Performance of the Continuous Performance Test among community samples. Schizophrenia Bulletin 1998a;24:163-74.

Chen WJ, Liu SK, Chang C-J, Lien Y-J, Chang Y-H, Hwu H-G. Sustained attention deficit and schizotypal personality features in nonpsychotic relatives of schizophrenic patients. American Journal of Psychiatry 1998b;155:1214-20.

Chen WJ, Chang C-H, Liu SK, Hwang TJ, Hwu H-G. Collaborators from the Multidimensional Psychopathology Group Research Project. Sustained attention deficits in nonpsychotic relatives of schizophrenic patients: a recurrence risk ratio analysis. Biological Psychiatry 2004;55:995-1000.

Cheng JJ, Ho H, Chang CJ, Lan SY, Hwu HG. Positive and Negative Syndrome Scale (PANSS): establishment and reliability study of a Mandarin Chinese language version. Chinese Psychiatry (Taipei) 1996;10:251-8.

Cornblatt BA, Keilp JG. Impaired attention, genetics, and the pathophysiology of schizophrenia. Schizophrenia Bulletin 1994;20:31-46.

Cornblatt B, Obuchowski M, Schnur B, O'Brien JD. Attention and clinical symptoms in schizophrenia. Psychiatric Quarterly 1997;68:343-59.

Cosway R, Byrne M, Clafferty R, Hodges A, Grant E, Morris J, et al. Sustained attention in young people at high risk for schizophrenia. Psychological Medicine 2002;32:277-86.

Davies DR, Parasuraman R. The Psychology of Vigilance. London: Academic Press; 1982.

Egan MF, Goldberg TE, Gscheidle T, Weirich M, Bigelow LB, Weinberger DR. Relative risk of attention deficits in siblings of patients with schizophrenia. American Journal of Psychiatry 2000;157:1309-16.

Epstein JI, Keefe RSE, Roitman SL, Harvey PD, Mohs RC. Impact of neuroleptic medications on continuous performance test measures in schizophrenia. Biological Psychiatry 1996;39:902-5.

Finkelstein JRJ, Cannon TD, Gur RE, Gur RC, Moberg P. Attentional dysfunctions in neuroleptic-naive and neuroleptic-withdrawn schizophrenic patients and their siblings. Journal of Abnormal Psychology 1997;106:203-12.

Flashman LA, Green MF. Review of cognition and brain structure in schizophrenia: profiles, longitudinal course, and effects of treatment. Psychiatric Clinics of North America 2004;27:1-18.

Guillem F, Bicu M, Bloom D, Wolf MA, Desautels R, Lalinec M, et al Neuropsychological impairments in the syndromes of schizophrenia: a comparison between different dimensional models. Brain \& Cognition 2001;46:153-9.

Heaton RK, Gladsjo JA, Palmer BW, Kuck J, Marcotte TD, Jeste DV. Stability and course of neuropsychological deficits in schizophrenia. Archives of General Psychiatry 2001;58:24-32.

Hill SK, Schuepbach D, Herbener ES, Keshavan MS, Sweeney JA. Pretreatment and longitudinal studies of neuropsychological deficits in antipsychotic-naive patients with schizophrenia. Schizophrenia Research 2004;68:49-63.

Hong KS, Kim JG, Koh HJ, Koo MS, Kim JH, Lee D, et al. Effects of risperidone on information processing and attention in first-episode schizophrenia. Schizophrenia Research 2002;53:7-16.

Hwu H-G, Chen C-H, Hwang T-J, Liu C-M, Cheng JJ, Lin S-K, et al. Symptom patterns and subgrouping of schizophrenic patients: significance of negative symptoms assessed on admission. Schizophrenia Research 2002;56:105-19.

Kay SR, Fiszbein A, Opler LA. The positive and negative syndrome scale (PANSS) for schizophrenia. Schizophrenia Bulletin 1987;13:261-76.

Keri S, Janka Z. Critical evaluation of cognitive dysfunctions as endophenotypes of schizophrenia. Acta Psychiatrica Scandinavica 2004;110:83-91.

Kremen WS, Seidman LJ, Faraone SV, Toomey R, Tsuang MT. The paradox of normal neuropsychological function in schizophrenia. Journal of Abnormal Psychology 2000;109:743-52. 
Laurent A, Biloa-Tang M, Bougerol T, Duly D, Anchisi AM, Bosson JL, et al. Executive/attentional performance and measures of schizotypy in patients with schizophrenia and in their nonpsychotic first-degree relatives. Schizophrenia Research 2000;46:269-83.

Lawrie SM, Byrne M, Miller P, Hodges A, Clafferty RA, Cunningham Owens DG, Johnstone EC. Neurodevelopmental indices and the development of psychotic symptoms in subjects at high risk of schizophrenia. British Journal of Psychiatry 2001;178:524-30.

Leask SJ, Done DJ, Crow TJ. Adult psychosis, common childhood infections and neurological soft signs in a national birth cohort. British Journal of Psychiatry 2002;181:387-92.

Lewis DA, Levitt P. Schizophrenia as a disorder of neurodevelopment. Annual Review of Neuroscience 2002;25:409-32.

Liu SK, Hwu H-G, Chen WJ. Clinical symptom dimensions and deficits on the Continuous Performance Test in schizophrenia. Schizophrenia Research 1997;25:211-9.

Liu SK, Chen WJ, Chang C-J, Lin H-N. Effects of atypical neuroleptics on sustained attention deficits in schizophrenia: a trial of risperidone versus haloperidol. Neuropsychopharmacology 2000;22:311-9.

Liu SK, Chiu C-H, Hwang T-J, Chang C-J, Hwu HG, Chen WJ. Deficits in sustained attention in schizophrenia and affective disorders: Stable versus state-dependent markers. American Journal of Psychiatry 2002;159:975-82.

Mass R, Schoemig T, Hitschfeld K, Wall E, Haasen C. Psychopathological syndromes of schizophrenia: evaluation of the dimensional structure of the positive and negative syndrome scale. Schizophrenia Bulletin 2000;26:167-77.

Mirsky AF, Yardley SL, Jones BP, Walsh D, Kendler KS. Analysis of the attention deficit in schizophrenia: A study of patients and their relatives in Ireland. Journal of Psychiatric Research 1995;29:23-42.

Muthen LK, Muthen B. Mplus User's Guide, Version 3.13. Los Angeles: Muthen \& Muthen, 2005.

Muthen B, Brown CH, Masyn K, Jo B, Khoo ST, Yang CC, et al. General growth mixture modeling for randomized preventive interventions. Biostatistics 2002;3:459-75.

Nieuwenstein MR, Aleman A, de Haan EH. Relationship between symptom dimensions and neurocognitive functioning in schizophrenia: a meta-analysis of WCST and CPT studies. Journal of Psychiatric Research 2001;35:119-25.

Nuechterlein KH, Dawson ME. Information processing and attentional functioning in the developmental course of schizophrenic disorders. Schizophrenia Bulletin 1984;10:160-203.

Nuechterlein KH, Edell ES, Norris M, Dawson ME. Attentional vulnerability indicators, thought disorder, and negative symptoms. Schizophrenia Bulletin 1986;12:408-26.

Nuechterlein KH, Dawson ME, Gitlin M, Ventura J, Goldstein MJ, Snyder KS, et al. Developmental processes in schizophrenic disorders: longitudinal studies of vulnerability and stress. Schizophrenia Bulletin 1992;18:387-425.
Nuechterlein KH, Dawson ME, Green MF. Information-processing abnormalities as neuropsychological vulnerability indicators for schizophrenia. Acta Psychiatrica Scandinavica 1994;90: 71-9.

Nurnberger Jr JI, Blehar MC, Kaufmann CA, York-Cooler C, Simpson SG, Harkavy-Friedman J, Severe JB, Malaspina D, Reich T, collaborators from the NIMH Genetics Initiative. Diagnostic Interview for Genetic Studies: Rationale, unique features, and training. Archives of General Psychiatry 1994;51:849-59.

Obiols JE, Serrano F, Caparros B, Subira S, Barrantes N. Neurological soft signs in adolescents with poor performance on the continuous performance test: markers of liability for schizophrenia spectrum disorders? Psychiatry Research 1999;86:217-28.

Olbrich R, Kirsch P, Pfeiffer H, Mussgay L. Patterns of recovery of autonomic dysfunctions and neurocognitive deficits in schizophrenics after acute psychotic episodes. Journal of Abnormal Psychology 2001;110:142-50.

Palmer BW, Heaton RK, Paulsen JS, Kuck J, Braff D, Harris MJ, et al. Is it possible to be schizophrenic yet neuropsychologically normal? Neuropsychology 1997;11:437-46.

Roitman SEL, Keefe RSE, Harvey PD, Siever LJ, Mohs RC. Attentional and eye tracking deficits correlate with negative symptoms in schizophrenia. Schizophrenia Research 1997;26:139-46.

Ross DE, Kirkpatrick B, Karkowski LM, Straub RE, MacLean CJ, O'Neill FA, et al. Sibling correlation of deficit syndrome in the Irish study of high-density schizophrenia families. American Journal of Psychiatry 2000;157:1071-6.

Rosvold HE, Mirsky AF, Sarason I, Bransome Jr ED, Beck LH. A continuous performance test of brain damage. Journal of Consulting Psychology 1956;20:343-50.

Saoud M, d'Amato T, Gutknecht C, Triboulet P, Bertaud J-P, MarieCardine M, et al. Neuropsychological deficit in siblings discordant for schizophrenia. Schizophrenia Bulletin 2000;26:893-902.

Stratta P, Daneluzzo E, Bustini M, Prosperini P, Rossi A. Processing of context information in schizophrenia: relation to clinical symptoms and WCST performance. Schizophrenia Research 2000;44:57-67.

Strauss ME. Relations of symptoms to cognitive deficits in schizophrenia. Schizophrenia Bulletin 1993;19:215-31.

Swets JA. The relative operating characteristic in psychology: A technique for isolating effects of response bias finds wide use in the study of perception and cognition. Science 1973;182:990-1001.

Tamminga CA, Buchanan RW, Gold JM. The role of negative symptoms and cognitive dysfunction in schizophrenia outcome. International Clinical Psychopharmacology 1998;13(Suppl):S21-6.

Tsuang H-C, Lin S-H, Liu SK, Hsieh M-H, Hwang TJ, Liu C-M, et al. More severe sustained attention deficits in nonpsychotic siblings of multiplex schizophrenia families than in those of simplex ones. Schizophrenia Research, in press. Available online 5 June 2006. 